\title{
NUEVO FRAGMENTO DE INSCRIPCIÓN PROCEDENTE DE VELEIA (IRUÑA DE OCA, ÁLAVA) CON POSIBLE EXPRESIÓN DE UN MAGISTRADO LOCAL ${ }^{1}$
}

\author{
NEW FRAGMENT OF AN INSCRIPTION \\ FROM VELEIA (IRUÑA DE OCA, ÁLAVA) \\ WITH A POSSIBLE EXPRESSION OF A LOCAL MAGISTRATE
}

\author{
Pilar Ciprés, Julio NúÑEz y Joaquín Gorrochategui \\ $U P V / E H U$ \\ pilar.cipres@ehu.es \\ julio.nunez@ehu.es \\ joaquin.gorrochategui@ehu.es
}

DOI: $10.1387 /$ veleia.14997

\begin{abstract}
Resumen: presentamos un nuevo fragmento de inscripción procedente del yacimiento romano de Veleia (Iruña de Oca, Álava). La inscripción fue descubierta durante los trabajos arqueológicos realizados en el cubo oeste de la puerta sur de la muralla, donde había sido reutilizada como sillar. El fragmento contiene parte de un epígrafe con una posible mención a un magistrado local. Si la hipótesis de lectura es correcta, se trataría del primer testimonio sobre la existencia de duumviri en la ciuitas Veleianorum.

Palabras clave: Veleia, epigrafía latina, ciuis romanus, IIvir, flamen.

Abstract: We present a new fragment of an inscription from the Roman site of Veleia (Iruña de Oca, Álava). The inscription was found while carrying out archaeological work on the western tower of the southern door of the wall, where it had been used as an ashlar. The fragment records part of an epigraph with a possible mention of a local magistrate. If our interpretation hypothesis is correct, it would be the first testimony of the existence of duumviri in the "ciuitas Veleianorum».

Keywords: Veleia, Latin epigraphy, ciuis romanus, IIvir, flamen.
\end{abstract}

Recibido: 07-07-2015

Definitivo: 10-07-2015

1 Este trabajo ha sido realizado en el marco de los diferentes proyectos de investigación en los que participan los autores: 1) HAR2011-27431/HIST del MICINN y GI IT760-13 del Gobierno Vasco; 2)
Ejecución del Plan Director Iruña-Veleia 2010-2020 DFA; HAR2011-23445 de MICINN 3) FFI201236069-C03-01 del MINECO y GI IT698-13 del Gobierno Vasco. 


\section{INTRODUCCIÓN}

El descubrimiento de este nuevo epígrafe «veleiense» que presentamos se produjo durante una de las actuaciones arqueológico-arquitectónicas planeadas para el año 2014 dentro del marco del Plan Director ${ }^{2}$. Su objetivo era la consolidación y conservación del cubo amurallado que defiende, por el oeste, la única puerta bien documentada, hasta el momento, que permitía el acceso más directo al denominado «oppidum de Iruña» desde el sur, es decir, desde la zona amurallada más visible en la actualidad. Esta puerta se abría al conocido como iter XXXIV "De Hispania in Aquitaniam ab Asturica Burdigalam», descrito en el Itinerario de Antonino, una de cuyas mansiones, Veleia, fue precisamente este enclave urbano, cuya identificación quedó confirmada con el hallazgo reciente de otro epígrafe (Núnez, Martínez, Ciprés y Gorrochategui 2012).

Tras intervenciones anteriores en el s. XIX y comienzos del s. Xx, es importante la realizada por G. Nieto entre los años 1949 y 1954, resumida en su memoria El oppidum de Iruña publicada en 1958. Su actuación se centró fundamentalmente en el frente sur del oppidum con la intención de hacer visible su monumentalidad, excepcional en esos momentos en el entorno. Esta situación no pasó desapercibida para Nieto quien concentró sus mayores esfuerzos — quizás no económicos pero sí "físicos» - en la limpieza ímproba de un tipo de ruina similar a la que en la actualidad resulta visible en amplios tramos de la muralla y que podríamos definir como taludes de piedra suelta y tierra. Sus trabajos se centraron en la cara sur de las murallas, sin duda la más sugerente desde el punto de vista monumental, poniendo al descubierto una realidad material más compleja de lo que se hubiese podido imaginar a partir de los datos previos a su intervención. Durante sus trabajos se recuperaron entre los escombros varias inscripciones, entre ellas las estelas recogidas en su memoria $^{3}$, y en su mayor parte incomprensiblemente perdidas.

\section{Circunstancias del hallazgo}

Como hemos dicho, los trabajos en el cubo oeste de la puerta situada en la muralla sur comenzaron a mediados del mes de octubre de 2014 con el objetivo de llevar a cabo su consolidación. La inscripción que presentamos fue descubierta entre los días 15 y 17 de este mes durante la limpieza previa a la conservación arquitectónica.

El epígrafe, sobre el que no existe ninguna noticia anterior a este momento, se encontraba fácilmente accesible, tan solo cubierto por la argamasa disuelta y la tierra acumulada — desde que la estructura de la puerta fuera descubierta durante los trabajos llevados a cabo por G. Nieto hace más de 50 años-.

2 El Plan director 2010-2020 para Iruña-Veleia, se planificó en el año 2009 y se presentó en 2010, si bien su ejecución se ha visto limitada por una parte y prolongada por otra.

3 Nieto 1958, 208-209, 213, 216 con fotos $=A E$ 1965, 61; HAEp 977, 978, 1777, 1781 y 1780; Elorza
1967, n. ${ }^{\circ} 73,74$ y 75 (este último número recoge varios fragmentos). A estas habría que ańadir la conocida como Estela del Jinete, anepígrafa, que fue extraída del muro interno del mismo cubo occidental de la puerta sur, dejando huellas estratigráficas que hemos podido reconocer ahora. 


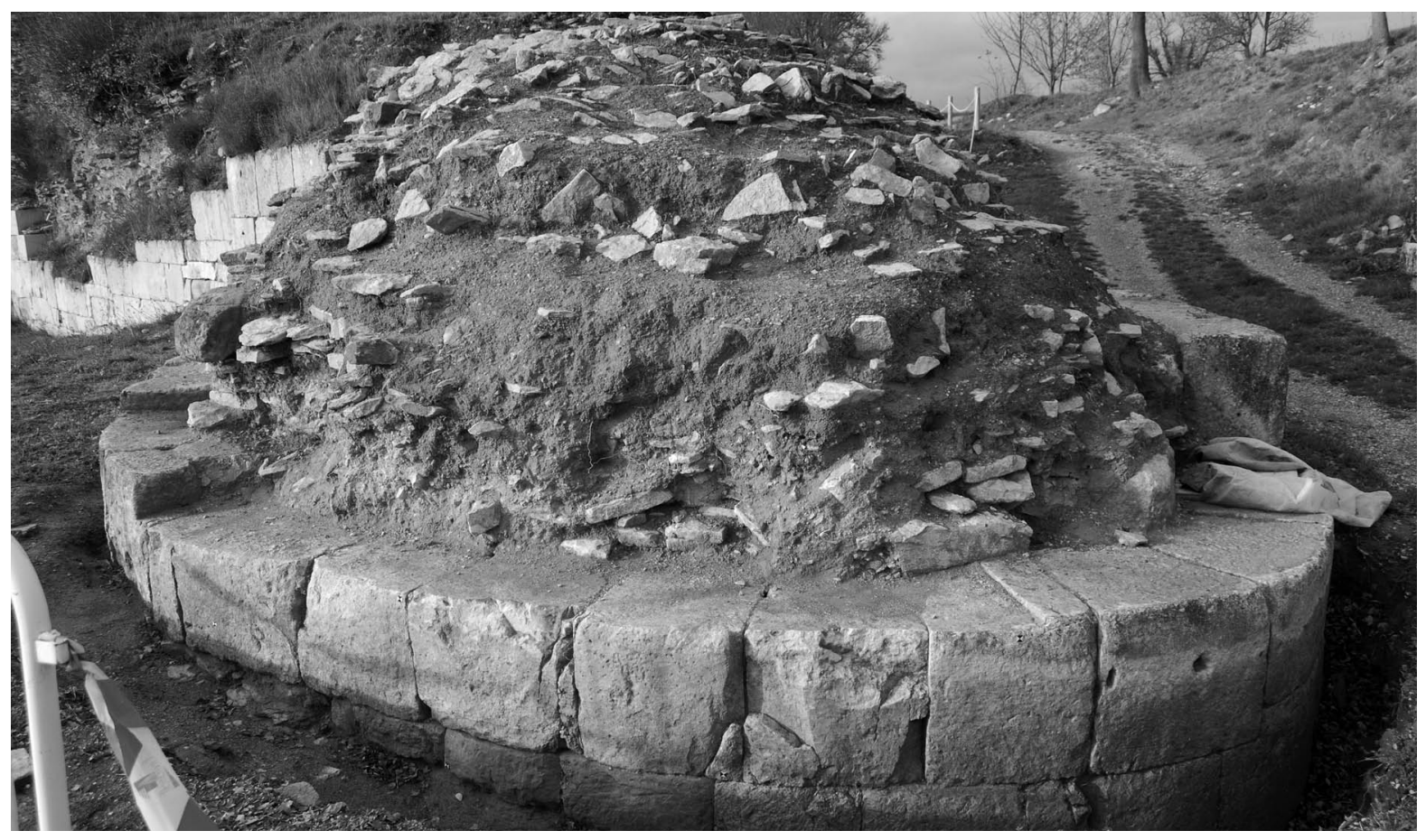

Figura I. Cubo oeste de la puerta sur

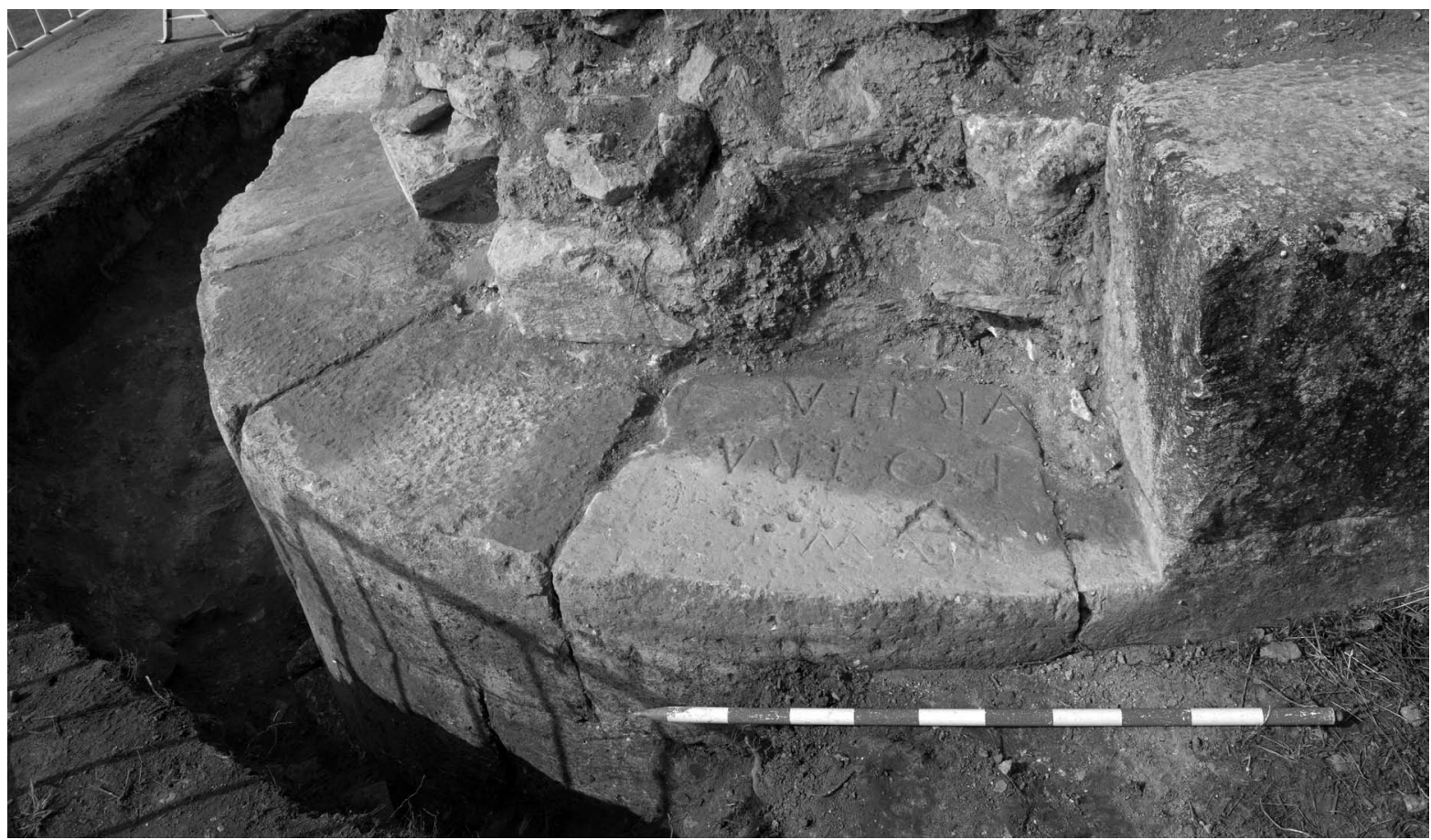

Figura 2. Localización en la pared exterior del cubo 


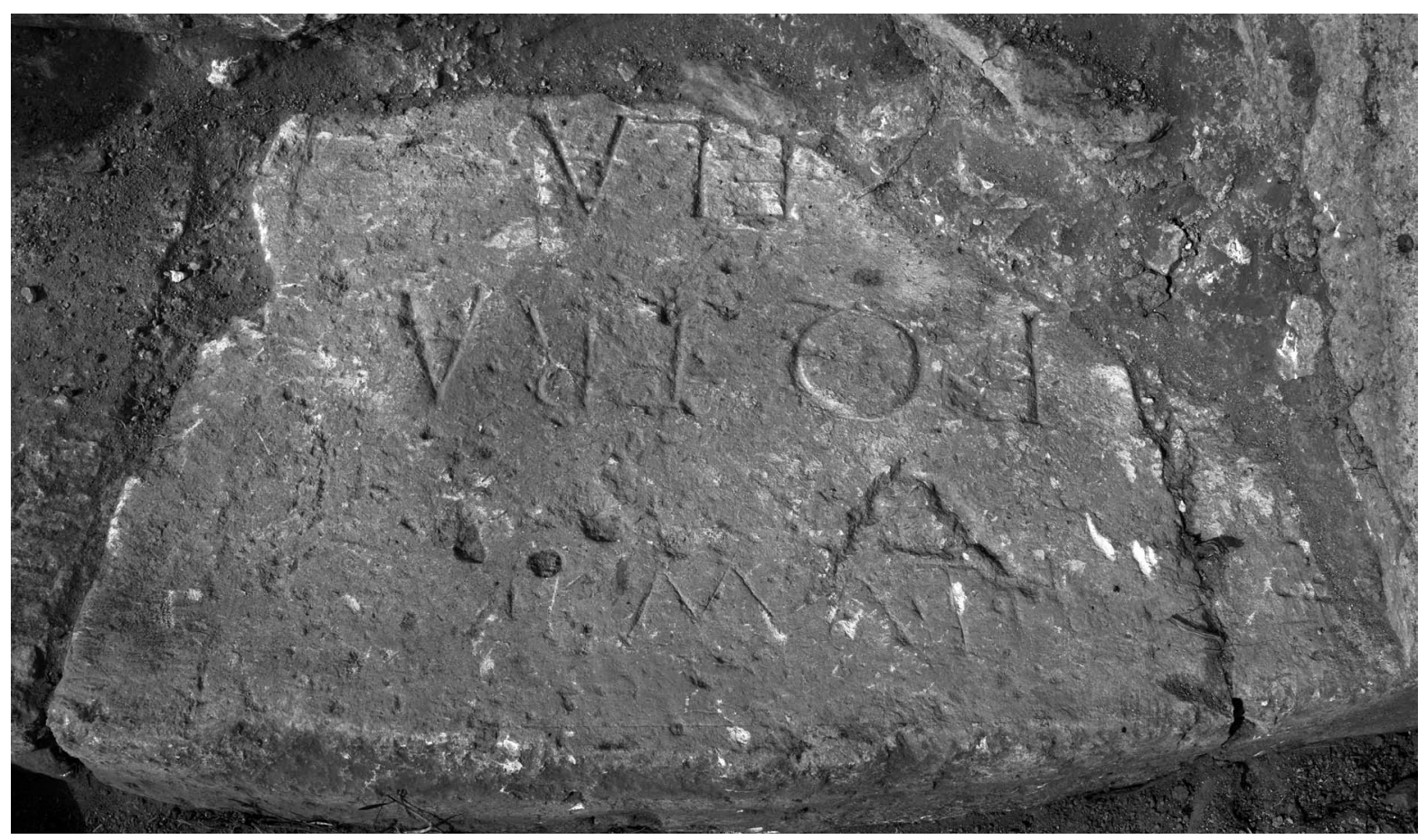

Figura 3. Detalle del hallazgo de la pieza

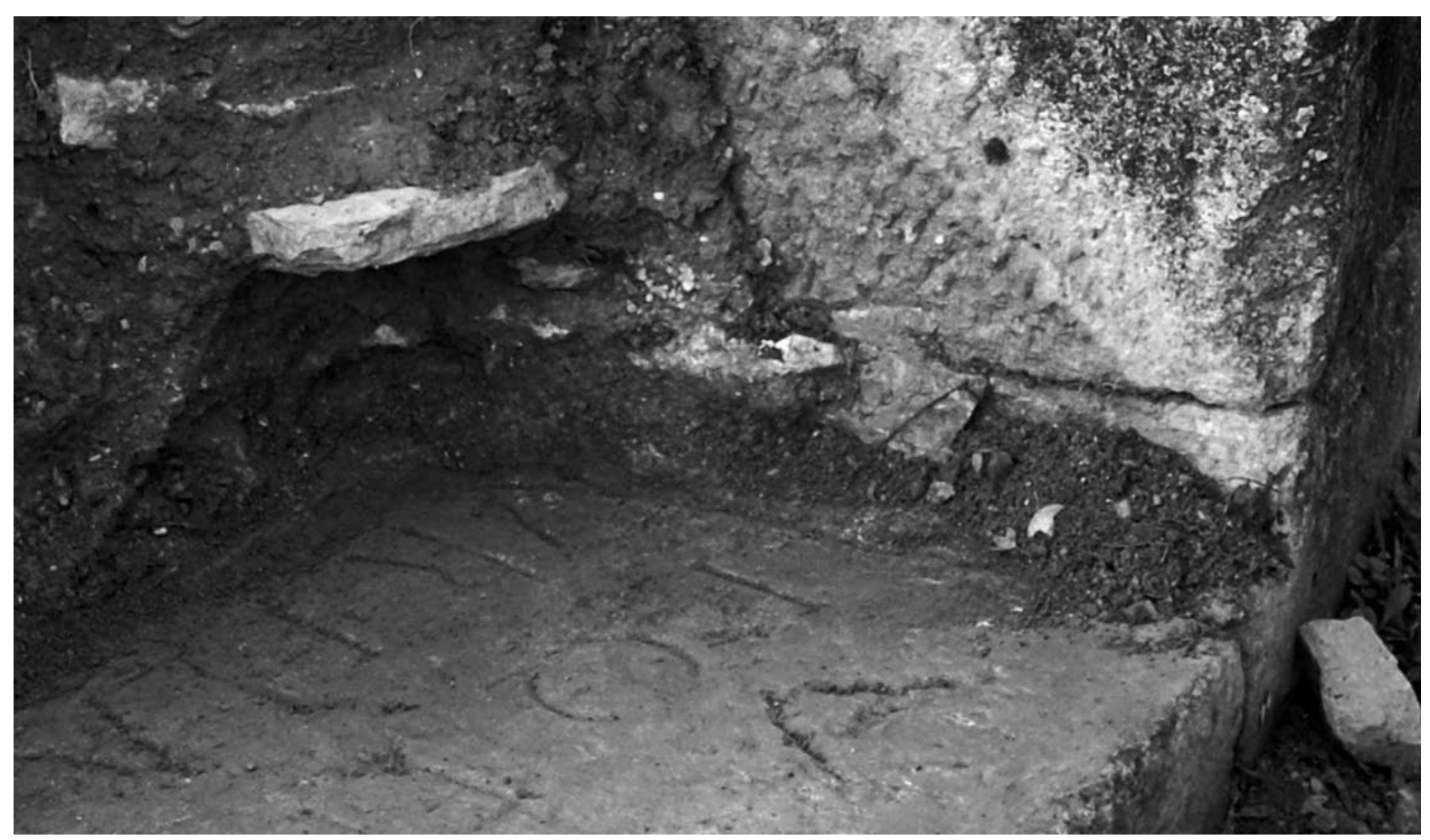

Figura 4. El epigrafe en fase de limpieza 
Una vez convenientemente documentada y realizada la autopsia ${ }^{4}$ se decidió por todas las partes implicadas en la conservación del patrimonio y en concreto la Comisión de Seguimiento del Plan Director de Iruña-Veleia, que la mejor solución para su preservación era su conservación in situ, cubierta con un nuevo bloque superior para protegerla de la erosión ${ }^{5}$.

\section{Soporte}

El fragmento corresponde a la parte derecha de un bloque, que ha sido recortado para su reutilización como sillar en la construcción del cubo oeste de la puerta sur, donde fue localizado en la segunda hilada de la pared exterior de la torre. Presenta un aspecto trapezoidal para adaptarse al diseńo del cubo, cuya planta no es semicircular sino ligeramente elíptica, por lo que el lado superior, colocado formando parte de la cara exterior de la pared, ha sido retallado de forma ligeramente curva para adecuarse al trazado de la misma. Por su parte el lado inferior, orientado hacia el interior, ha sido recortado de forma irregular. La anchura máxima del soporte es de $81,35 \mathrm{~cm}$ en la parte superior de la cara frontal y de $69,1 \mathrm{~cm}$ en la inferior, mientras que la altura oscila entre los $59,2 \mathrm{~cm}$ en el lado izquierdo y los $56,9 \mathrm{~cm}$ en el derecho. El grosor parece corresponder al original de la pieza. Aunque no podemos establecer con seguridad sus dimensiones totales, las medidas del fragmento y la posible restitución del texto hacen pensar en un bloque entre $120 \mathrm{o} 150 \mathrm{~cm}$ de ancho con una altura, tal vez, no inferior a $75 \mathrm{~cm}$. si el ordinator ha respetado en el tercer renglón el módulo de las letras y ha mantenido el interlineado de $6,8 \mathrm{~cm}$.

El bloque, tal vez un arquitrabe, podría formar parte de un monumento funerario o de un edificio. No se trata del primer testimonio epigráfico procedente de Veleia vinculado a una construcción edilicia o a un monumento de mayores dimensiones. Junto a los dos bloques descubiertos por J. de Verástegui a principios del siglo xx y recogidos, por primera vez, por F. Baraibar, con tres letras de $21 \mathrm{~cm}$ pertenecientes a un epígrafe de carácter monumental (Baraibar 1912, 8 = HAEp 2544), debemos añadir la inscripción dedicada a M(arco) Lucretio/ Fraterno Plinthlas pater $(H A E p, 978)^{6}$ grabada sobre un bloque paralelepípedo de $23 \mathrm{~cm}$ de altura por $100 \mathrm{de}$ ancho ${ }^{7}$, también reutilizado en la construcción de la muralla, más concretamente «en la cara interior de la muralla junto a la puerta» (Nieto 1958, 126-127). Por sus características esta última inscripción parece identificarse con un epitafio que debió formar parte de un monumento funerario o un mausoleo.

El fragmento en la actualidad contiene tres textos sin ninguna relación entre sí: la inscripción original de la que se han conservado parte de dos renglones epigráficos y otras dos posteriores, que describimos en el apéndice final, ambas localizadas sobre la primera en disposición inversa.

\footnotetext{
4 Tras los trabajos documentación arqueológica, la autopsia de la inscripción se llevó a cabo el día 26 de noviembre de 2014 .

5 Se agradece al arquitecto Oscar Reinares su opinión autorizada a este respecto.

6 El bloque mide 23 x $100 \mathrm{~cm}$ y la altura de las letras disminuye de forma progresiva de la línea 1 a la 3 (1. $1: 7 \mathrm{~cm} ; 1.2: 5 \mathrm{~cm}$ y $1.3: 3 \mathrm{~cm}$ ). En la actualidad al desgaste sufrido por la piedra debemos ańadir la des-
}

trucción de la parte central del epígrafe. Con anterioridad a ésta el bloque presentaba dos fracturas oblicuas dispuestas de arriba abajo y un desconche en su parte superior que cortaba por la mitad las letras de la línea 1 , tal y como se puede apreciar en la fotografía conservada (Nieto 1958, 215, con foto).

7 Nieto 1958, 215, con foto ; Elorza 1967, 157, 67 ; ILER, $4127=6808$. 


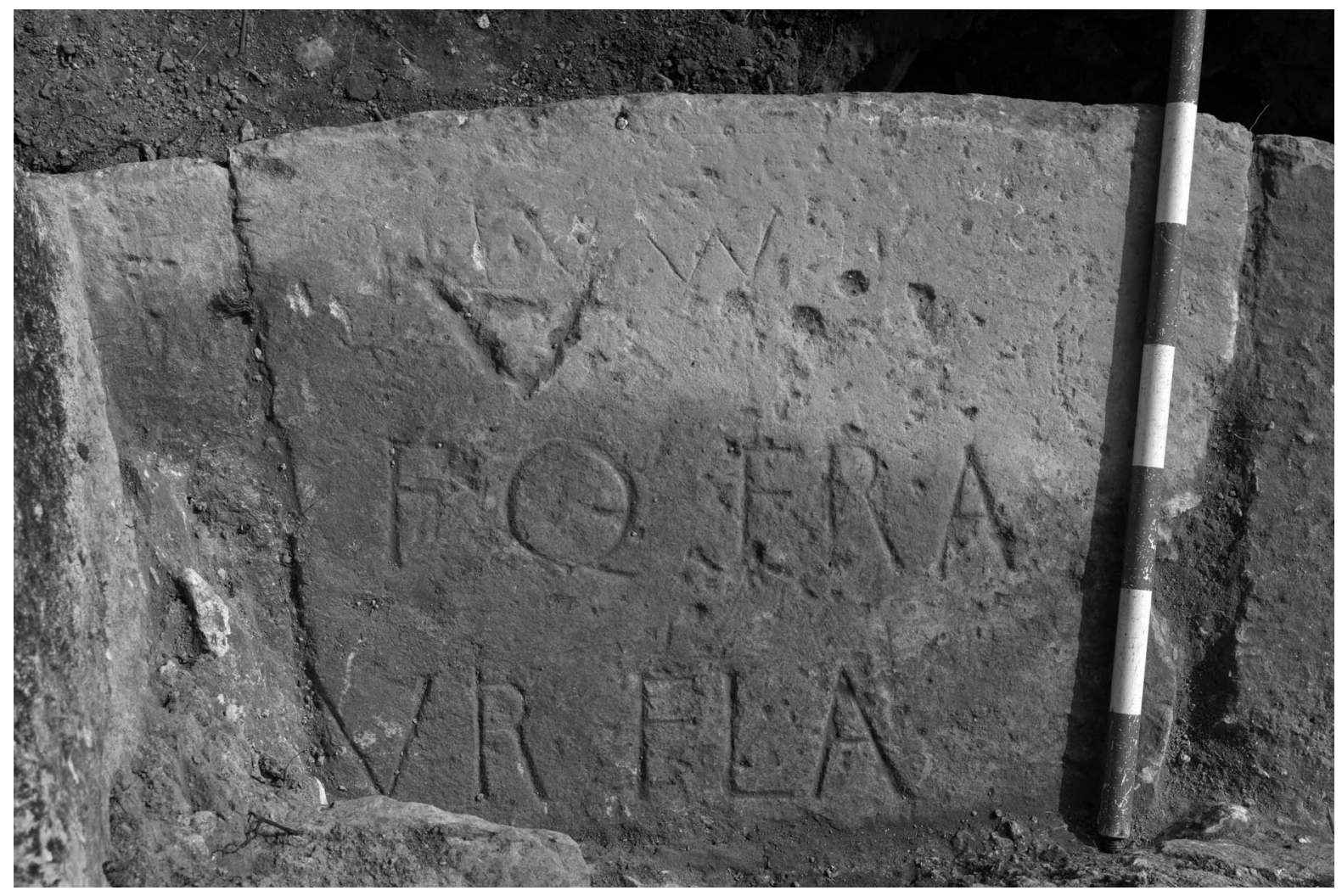

FIGURA 5. Imagen completa de la superficie grabada

\section{EPÍGRAFe I:}

$$
\begin{aligned}
& \text { [---]F Q FRA } \\
& \text { [---]V'IR' FLA } \\
& \text { [--- }
\end{aligned}
$$

Altura de las letras: línea 1: 10, $2 \mathrm{~cm}$; línea 2, R: 9,5; I: $12 \mathrm{~cm}$ (nexo IR) y el resto de las letras $10,2 \mathrm{~cm}$.

Interlineado: $6,8 \mathrm{~cm}$.

El campo epigráfico principal pudo ocupar toda la cara frontal del bloque, sin que se aprecien restos de algún elemento de delimitación. Su superficie aparece desgastada, con pequeños golpes y desconches que afectan a los trazos de las letras, pero que no impiden su identificación. Así sucede, por ejemplo, en la línea 1 junto a los trazos horizontales de la primera $\mathrm{F}$, en el extremo inferior de la segunda, en el asta vertical de la $\mathrm{R}$ o en la $\mathrm{A}$.

El texto ocupa prácticamente la mitad inferior de la cara frontal, quedando un amplio espacio entre la línea 1 y el borde superior, que alcanza los $25 \mathrm{~cm}$ sobre la primera $\mathrm{F}$ y los $29 \mathrm{~cm}$ sobre la A. No se aprecian restos de líneas guía, ni signos de interpunción. La separación entre las letras es 


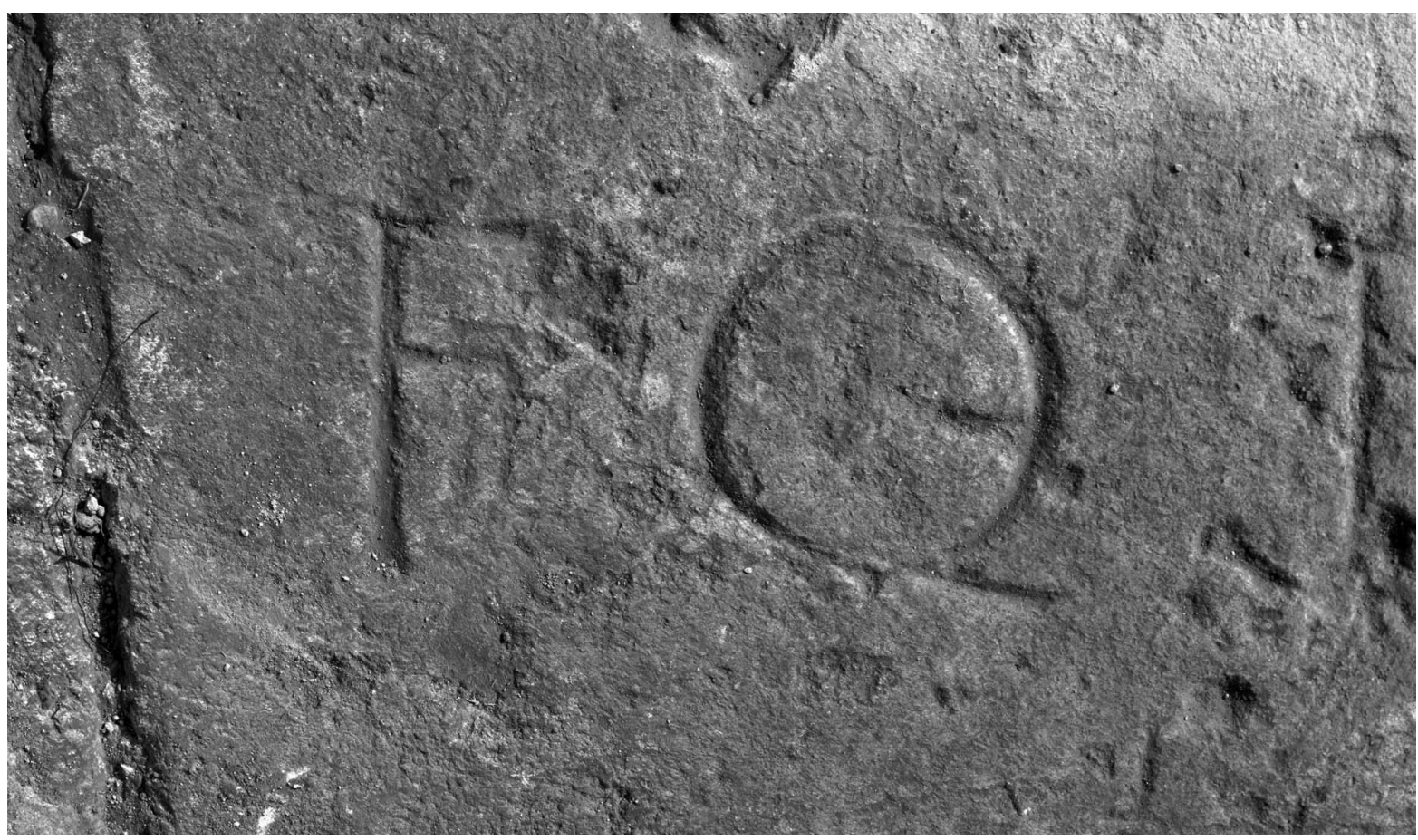

Figura 6a. Detalle del inicio de la línea 1

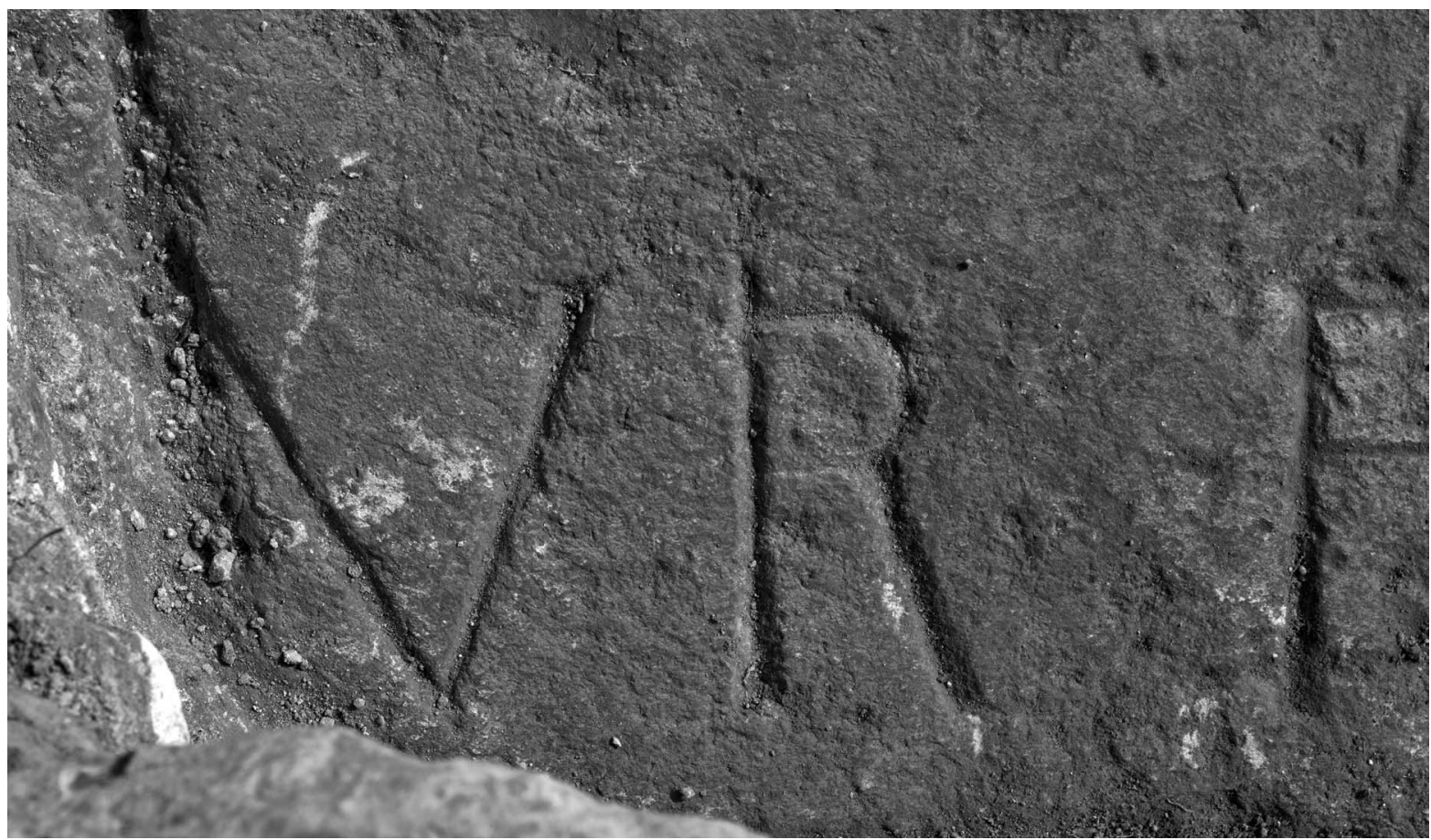

Figura 6в. Detalle del inicio de la línea 2 y nexo 
mayor entre palabras que dentro de cada palabra, aunque éstas se encuentren abreviadas. Así, por ejemplo, en la línea 1 entre la primera $F$ y la $Q$ hay un espacio de $5 \mathrm{~cm}$ y entre la $Q$ y la segunda $F$ de $8,1 \mathrm{~cm}$, mientras que en FRA la separación es de 2,7 y 2,4 cm respectivamente. Lo mismo sucede al inicio del renglón, donde no se observa ningún resto de la letra anterior a la $\mathrm{F}$, quedando un espacio de aproximadamente $7 \mathrm{~cm}$ sin grabar. En la línea 2 la distancia entre la $\mathrm{R}$ y $\mathrm{F}$ es de $6,6 \mathrm{~cm}$, mientras que entre la $\mathrm{V}$ y el nexo IR es de $3 \mathrm{~cm}$ y entre la $\mathrm{F}$ y la $\mathrm{L}$ de $2,5 \mathrm{~cm}$ y entre esta última y la A de $1,9 \mathrm{~cm}$.

Las letras corresponden a una capital cuadrada de buena factura. Tan solo la A del primer renglón parece presentar un grabado algo más irregular, que puede ser consecuencia del desgaste sufrido por la piedra. El grabado, biselado y con arista central, es bastante profundo, conservándose mejor en la línea 2. Con la excepción del nexo IR las letras presentan una altura uniforme de $10,2 \mathrm{~cm}$. Si atendemos al ductus, no hay diferencia entre trazos finos y gruesos si bien algunos trazos oblicuos y verticales se ensanchan ligeramente hacia la base reforzados por un pequeńo ápice en su extremo. La forma de las letras es bastante uniforme detectándose solo pequeñas diferencias. La A, que aparece siempre con el travesaño horizontal, en la línea 1 es algo más ancha en el extremo inferior $(7 \mathrm{~cm})$ que en la línea $2(6 \mathrm{~cm})$. La F muestra en todos los casos los dos trazos horizontales rectos —el inferior ligeramente alto respecto al asta vertical - y de idéntica longitud $(4 \mathrm{~cm})$ salvo en la primera $\mathrm{F}$ de la línea 1 donde el inferior es algo menor $(3,6 \mathrm{~cm})$. La $\mathrm{V}$ es muy abierta con una apertura máxima de $9 \mathrm{~cm}$. En la $\mathrm{R}$ el bucle es abierto con un tamaño inferior a la mitad del asta vertical. El trazo oblicuo es recto arrancando del extremo inferior del bucle. La Q se presenta como un círculo de $10 \mathrm{~cm}$ de diámetro, cuya ejecución no llega a ser perfecta y del que arranca un rabo recto de $4 \mathrm{~cm}$ de largo. En la línea 2 se ha utilizado un nexo IR, en el que la I sobresale en altura $(12 \mathrm{~cm})$ respecto a la $\mathrm{R}(9,5 \mathrm{~cm})$ y a las restantes letras. $\mathrm{L}$ ha sido realizada con el trazo horizontal recto de 4,7 cm.

La restitución del epígrafe resulta problemática debido a la extensión del texto conservado. Según la información aportada por este, el epígrafe debía constar, al menos, de tres líneas, de las que se habría conservado el final de los dos primeros renglones, aunque no podemos descartar con total seguridad la existencia de una línea anterior dado el espacio existente hasta el borde superior del bloque. En caso de que fuera así, deberíamos pensar en un renglón de menor extensión, que pudiera contener, por ejemplo, una palabra o las siglas DM, si estamos ante una inscripción funeraria. La ordinatio parece indicar que la línea 1 ocupaba una mayor extensión que la línea 2 . Aparentemente podríamos pensar que esta última habría sido centrada respecto a la anterior, sin embargo es muy probable que ambas pudieran haber sido alineadas a la izquierda, como sucede en otros ejemplos bien conocidos ${ }^{8}$. Aunque no podemos establecer con seguridad la extensión de los renglones epigráficos, los datos aportados por el texto parecen indicar que habría desaparecido aproximadamente la mitad izquierda de ambos renglones. Teniendo en cuenta las dimensiones del fragmento y el texto conservado, podemos sugerir dos restituciones hipotéticas para este epígrafe, que afectan en particular a la lectura de la línea 2.

Los datos indican que entre la primera línea y el comienzo de la segunda se habría grabado la identidad de un individuo portador de la ciudadanía romana. Su nombre reproduce el sistema onomástico romano del que se ha conservado parte de la indicación de la filiación, F(ilius), la abreviatura de la tribu $Q$ (uirina) y la parte inicial del cognomen, que debía desarrollarse completamente íntegro en el renglón siguiente. Así, pues, ha desaparecido el praenomen abreviado, el nomen y el

8 La inscripción de Iruña anteriormente citada presenta el texto tan solo alineado a la izquierda en las dos primeras líneas. El tamaño de las letras de buena factura disminuye progresivamente desde la línea 1 a la 3 (1.1: 7 $\mathrm{cm}$; $1.2: 5 \mathrm{~cm}$ y $1.3: 3 \mathrm{~cm})$. Destaca la utilización en la línea 2 del nexo NT en el nombre del padre. 
nombre del padre en la expresión de la filiación. Aunque no podemos identificar cada uno de estos elementos, es posible establecer algunas hipótesis a partir del contexto epigráfico documentado hasta el momento en Veleia. Dentro del conjunto de la epigrafía de la provincia de Álava, en Veleia y su entorno se concentra el mayor número de testimonios de ciudadanos romanos portadores de tria nomina, con o sin expresión de la filiación y mención de la tribu (Ciprés 2006, 94-97 y 120). Entre los nomina documentados se encuentran formas habituales en la epigrafía hispana, entre las que predomina Sempronius. Si bien en los tres testimonios procedentes de Veleia aparece desarrollado íntegramente (CIL II, 2930; HAEp 1778), en Alaiza y Ocariz lo hace abreviado en SEMP (CIL II, 2943; EE VIII, 168). Para esta inscripción podemos pensar en cualquier nomen latino expresado incluso de forma abreviada.

Respecto al cognomen este debe identificarse con el antropónimo latino Fraternus, atestiguado en Hispania (Abascal 1994, 372) y documentado en la epigrafía alavesa en la inscripción HAEp, 978 procedente de Iruña, anteriormente mencionada. En este caso volvemos a encontrar, como en los de Maternus, Paternus, Fuscus, Flauus/Flauos o Flaccus también documentados en la zona, la adopción de antroponimia latina que remite a la existencia de un componente indígena (Abascal 1984, 219-259).

En cuanto a la indicación de la filiación, que en el epígrafe aparece incompleta, la nomenclatura romana nos lleva a considerar la posibilidad de la mención del praenomen del padre abreviado o no, siguiendo prácticas atestiguadas en Iruña (HAEp 1778: [.] Sempro[ni] u[s] Materni f(ilius) Qui(rina tribu) Maternus; HAEp, 978 M. Lucretius Fraternus; HAEp, 1780: [.] [S]ulpici[us] Quir(ina tribu) [---]nus; CIL II, 2933: G. Fabrici[us] Fuscus; CIL II 2930: [Ma]rcus Sem[pron]ius L(uci) f(ilius) Q(uirina tribu) Fl[auus?]). Si tenemos en consideración todas las posibilidades documentadas en la epigrafía del entorno, en principio tampoco podemos desechar la posibilidad de una filiación a través del nombre único del padre o de su cognomen, tal y como atestiguan otras inscripciones de Iruña (HAEp 1778: [.] Sempro[ni]u[s] Materni f(ilius) Qui(rina tribu) Maternus), Margarita (CIL II 2928: M. Octauius Sabini f(ilius) Qui(rina tribu) Ca[lli]cus) y San Esteban de Treviño (CIL II 2929: M. Porcius Ausci fi(lius) Qui(rina tribu) Tonius)9.

Aunque la indicación de la tribus no es muy abundante en la epigrafía alavesa, la única atestiguada hasta el momento es la Quirina, documentada en seis ocasiones, cinco de ellas en Iruña ${ }^{10}$. Habitualmente abreviada bajo la forma QVIR, en la inscripción de Iruña CIL II 2930, actualmente desaparecida, viene expresada como en el epígrafe que presentamos con la inicial $Q:[M a] r c u s$ Sem [pron]ius L(uci) f(ilius) Q(uirina tribu) Fl[auus?] ${ }^{11}$. Su presencia nos hace pensar en una naturalización efectuada a partir de los flavios, sin que podamos establecer la vía de acceso.

Algo más problemática resulta la restitución íntegra de la línea 2. Aunque no podemos establecer la extensión exacta de cada renglón epigráfico, podrían plantearse dos hipótesis. Teniendo en cuenta el texto conservado en la segunda línea, podríamos pensar en la posibilidad de que [---]VIR FLA formaran parte del sistema onomástico de un segundo individuo presente en la inscripción, debiendo

9 Respecto al uso en la filiación del nombre único del padre o del cognomen vid. Ciprés 2006, 114-119.

10 En Iruña y su entorno próximo: $H A E p$ 1778, CIL II 2930, CIL II 2928; HEp 4, 2 у HAEp 1780. El otro testimonio se localiza en San Esteban de Treviño: CIL II 2929. Fuera de la provincia de Álava hallamos la mención a la tribu en Forua (Vizcaya), HAEp 228.
11 Aunque la expresión de la tribus comúnmente aparece bajo la forma abreviada $Q V I R$, no faltan testimonios en los que se utiliza la inicial: CIL II 2681, 2802 y 5700; $A E 1981$, 548; $A E 1987,615 \mathrm{a}$; $A E 1991$, 1073; ERPSoria 39; HEp 2, 457; IRPLeón 155; HEp 13, 946 y 955 . Es posible que en la inscripción HAEp 2524 de Angostina (Álava) la $Q$ de $M$. Vibius $Q$. Flauinus esté indicando la tribus. 
entender VIR como parte de la tribu [Q] VIR(ina) y FLA como parte de un cognomen del tipo Flauus o Flauinus. Si esto fuera así, la extensión del texto debería ser muy larga, faltando el praenomen, el nomen y, tal vez, la filiación. En este caso, llama la atención la distinta abreviatura utilizada para la expresión de la tribu Quirina en el mismo epígrafe (Vid. Fig. 6 B).

Sin embargo las letras conservadas en la línea 2, las posibles dimensiones del mismo y la práctica epigráfica documentada en otros ámbitos sugiere una segunda hipótesis para la restitución del texto, en nuestra opinión más plausible.

El fragmento permite considerar la posibilidad de que el epígrafe pertenezca a un ciudadano romano del que, tras indicar su nombre, habrían sido grabadas las funciones que desempeñó en vida. Las letras [---]VIR FLA /--- podrían corresponder a IIVIR y FLAMEN. Si esta posibilidad es correcta, el final de flamen habría sido grabado al comienzo de la $1.3^{12}$. La secuencia IIVIR FLAMEN, bien atestiguada en la epigrafía del imperio, en Hispania se documenta mayoritariamente en la Citerior, donde su presencia se concentra en la zona oriental (Tarraco, Barcino, Saguntum, etc.), siendo la inscripción $A E$ 1966, 185 procedente de Huesca el testimonio más próximo al que presentamos. Si en la línea 1 el nomen no es muy largo o está abreviado y la filiación viene expresada a través de la inicial de praenomen del padre, en la línea 2 solo se habrían grabado el final del cognomen y las funciones mencionadas. Por el contrario, la presencia en el primer renglón de un nomen extenso como Sempronius y/o de la filiación a través del nombre único del padre o de su cognomen, y considerando la posibilidad de que ambos renglones estuvieran alineados a la izquierda, dejaría espacio para algún elemento más antes del término IIVIR. Tampoco disponemos de datos que permitan reconstruir la extensión y el final del epígrafe ${ }^{13}$.

Según todo lo dicho la restitución del texto podría ser:

$$
\text { [---]f(ilius/-o) Q(uirina tribu) Fral[ternus/-o---II] uir/(-o) fla|[men/-mini---] }
$$

Si esta segunda hipótesis es acertada, la inscripción que presentamos constituiría el primer testimonio epigráfico de una magistratura y un sacerdocio de ámbito local en Veleia. Aunque no contamos con ningún otro testimonio que nos confirme la existencia de este tipo de funciones públicas de carácter político-administrativo y religioso en la ciuitas Veleianorum, sin embargo esta posible restitución está en consonancia con otros epígrafes y fragmentos procedentes del mismo yacimiento ${ }^{14}$, que parecen indicar una estructura organizativa próxima al modelo de funcionamiento de las comunidades con estatuto jurídico privilegiado, en este caso, debemos pensar que como consecuencia de la concesión del ius Latii ${ }^{15}$.

12 En CIL II, 3008 = CIL II, 5837 (La Puebla de Castro, Huesca) y en $A E 2011,549$ (Saelices, Cuenca), aunque se trata de inscripciones sobre un soporte diferente, el término flamen también ha sido grabado entre dos renglones epigráficos.

13 Es posible la mención del o los dedicantes (si los hubo) y/o la existencia de una fórmula final.

14 El fragmento de inscripción (HAEp 2541) con la fórmula honore contentus impensam remisit (Ortiz de Urbina 2009) y el ara dedicada a Mater Dea con la mención de Res Publica Veleianorum (Núñez, Martínez, Ciprés y Gorrochategui 2012). Las excavaciones arqueológicas realizadas en las últimas campañas han sacado a la luz un macellum público, cuya primera fase de construcción data del último cuarto del siglo i y con una gran transformación en el s. II.

15 La mención epigráfica a magistrados locales y/o flamines no es frecuente en este ámbito de la Provincia Hispania Citerior. En el caso de las magistraturas locales los testimonios son muy escasos limitándose a los aediles de Andelos, Mendigorría, Navarra, ( $A E$ 1989, $456=A E 2007,793)$ y los magistrados documentados en Lara de los Infantes, Burgos ( $A E$ 1981, 548 y $A E$ 1993, 1041). Respecto al flaminado local o provincial los testimonios más cercanos proceden de Astorga, Clunia y Segobriga. 
Los escasos datos proporcionados por el fragmento nos impiden determinar con seguridad la naturaleza del epígrafe así como su origen público o privado. Solamente queda sugerir algunas propuestas plausibles, a partir del texto fragmentario y de las características del soporte. El primer ámbito en el que se puede hallar una explicación es el funerario. Decantándonos en la posibilidad de que el bloque que nos ocupa perteneciese a un monumento funerario, podríamos atribuirlo básicamente a un par de tipos arquitectónicos teniendo en cuenta sus dimensiones y las consecuencias de su retalle. El primero de estos tipos, el más monumental, convendría a un mausoleo tipo templo que, en cualquier caso nos llevaría a pensar en un simple templete «in antis». Por otro lado, una segunda suposición dada la posición actual del epígrafe, sería la de asociarlo a un mausoleo turriforme, tipología muy bien distribuida en la Península Ibérica, donde los encontramos en los lugares más diversos: Ampurias, Tarraco, Corduba, etc. ${ }^{16}$

En ese caso estaríamos ante el texto que indicaba la identidad del difunto, expresado en nominativo o en dativo, si en la parte inferior se hiciera mención del o los dedicantes. La posibilidad de lectura que acabamos de mencionar (en dativo) con la presencia de las funciones locales desempeñadas sería compatible también con que la función del epígrafe fuera honorífica, aunque el espesor del fragmento conservado no apoye que se tratara de un cubo o dado de inscripción honorífica al uso. Sin embargo no podemos descartar la posibilidad de que pudiera tratarse de un epígrafe en honor de un personaje destacado de la comunidad realizado en la tumba, en similitud con los pedestales y estatuas de carácter honorario ubicadas, no en el foro, sino en las necrópolis o en espacios domésticos (Melchor 2006). Por último, el texto y el soporte nos hacen considerar la posibilidad de que el texto sea de naturaleza edilicia, es decir, inscrito con la finalidad de señalar la construcción de algún edificio, o mejoras y ampliaciones en alguno previo, por parte del magistrado en cuestión, sin que podamos precisar si se trataba de una acción evergética a título privado o en virtud de su cargo.

Es una lástima que ninguna de las posibilidades someramente mencionadas, o alguna otra, no pueda ser comprobada por el infortunio de la pérdida del resto de la inscripción, que viene a sumarse a las carencias de información relativas a la denominación personal y detalles de la magistratura. Pero al tiempo que nos lamentamos de este infortunio, nos alegramos de que la buena fortuna nos haya deparado la atestiguación en Veleia de la máxima magistratura local en una comunidad regida por el derecho latino a partir, probablemente, del periodo Flavio (Ortiz de Urbina 2000).

\section{Cronología}

El primero en plantear un panorama cronológico para la muralla fue el propio Nieto, quien planteaba, siguiendo los argumentos de su época, que la misma fue construida a finales del siglo III, más concretamente hacia el 284 d.C., y estaría en funcionamiento hasta el siglo v proponiendo la fecha del 446 d.C. La cronología manejada desde entonces para esta construcción no ha variado mucho desde el punto de vista historiográfico, y la mayoría de los autores que se han referido a ella coinciden en situar su construcción a finales del siglo III, o comienzos del IV, y su abandono en el v. Como sabemos el fragmento sería obligatoriamente anterior a estas fechas. Aunque carecemos de elementos internos que nos permitan una datación más precisa del epígrafe, el contexto histó-

16 Sobre este tema vid. el reciente trabajo de RuizAlcalde y Charquero 2014, donde se pueden encontrar numerosas referencias bibliográficas. 
rico y la mención de la tribu Quirina llevan a considerar una cronología a partir de los Flavios, posiblemente del s. II d.C.

\section{ApÉNDICE}

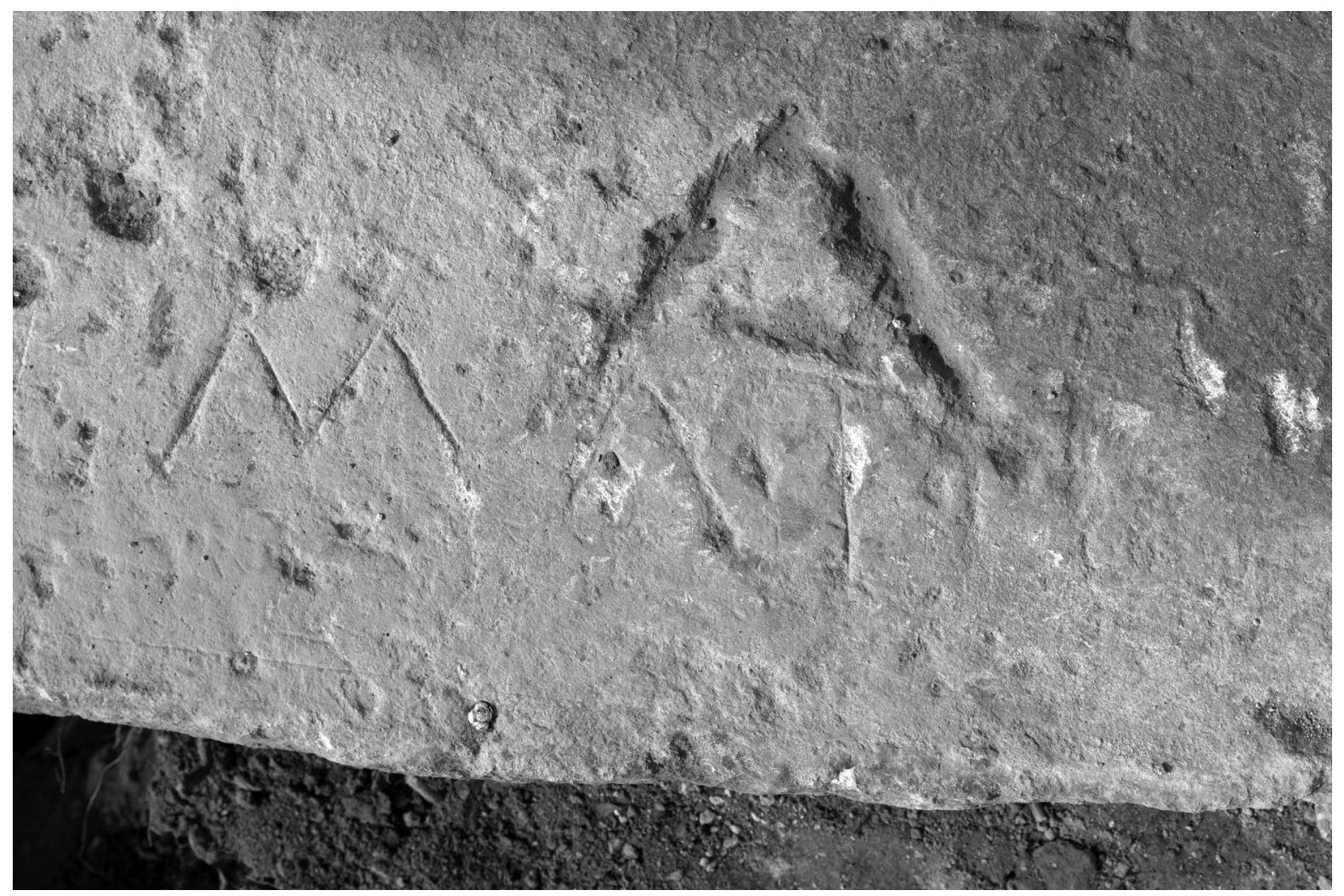

Figura 7. Epigrafes 2 y 3

\section{Epigrafe 2}

\section{MAT}

Este epígrafe ha sido grabado en disposición inversa sobre el primero a una distancia del borde superior entre 7 y $9 \mathrm{~cm}$. Las letras han sido grabadas de forma tosca con un trazo irregular, fino y no muy profundo. La $\mathrm{M}$ es abierta con los trazos oblicuos; su altura es de $5,4 \mathrm{~cm}$ con el izquierdo algo más largo $(6,7 \mathrm{~cm})$. La A no tiene travesaño y la longitud de sus trazos varía, siendo en el primero de $5 \mathrm{~cm}$ y en el segundo de $7 \mathrm{~cm}$. La T con una altura de $7,2 \mathrm{~cm}$ presenta el trazo horizontal de ca. $5 \mathrm{~cm}$ de largo desplazado hacia la izquierda y en disposición ligeramente oblicua. Después de la $\mathrm{T}$ aparecen dos pequeñas incisiones que no forman parte de la inscripción. 


\section{Epigrafe 3}

\section{A}

Grabada directamente sobre la inscripción original, pero en sentido inverso, es decir de este a oeste, lo que nos lleva a pensar en que fue grabada una vez instalado el bloque retallado en su nueva posición. Se trata de una única letra, seguramente una A muy abierta $(14 \mathrm{~cm}$ de ancho en la parte inferior), en la que el travesaño corto no llega a unirse al trazo izquierdo. Su altura es de $10 \mathrm{~cm}$ y ha sido tallada de forma muy burda con un instrumento bastante grosero que dejó en todos sus vértices líneas dentadas. Tratándose de una única letra y por las deficiencias de su grabado, cabe pensar en una simple marca de cantero, obviamente del que retalló el bloque para su inserción en la muralla.

\section{BibliografíA}

Abascal Palazón, J. M., 1984, «Los cognomina de parentesco en la Península Ibérica. A propósito del influjo romanizador en la onomástica», Lucentum 3, 219-259.

—, 1994, Los nombres personales en las inscripciones latinas de Hispania, Madrid-Murcia: Universidad Complutense de Madrid y Universidad de Murcia.

Baraibar, F., 1912, Museo incipiente. Suplemento a la memoria del curso 1911 a 1912 en el Instituto General y Técnico de Vitoria, Madrid.

Ciprés, P., 2006, «La onomástica de las inscripciones romanas del País Vasco. Estructura del nombre personal y estatuto jurídico», Veleia 23, 85-128.

Elorza, J. C., 1967, «Ensayo topográfico de epigrafía romana alavesa», EAA 2, 119-186.

Melchor, E., 2006, "La ubicación de los homenajes estatuarios en las ciudades de la Bética», CCG 17, 201-211.

Nieto, G., 1958, Oppidum de Iruña (Álava): memoria de las excavaciones, Vitoria: Consejo de Cultura de la Diputación Foral de Álava .

Núñez, J., Martínez, D., Ciprés, P. y Gorrochategui, J., «Nueva ara dedicada a Mater Dea procedente de Veleia (Iruña de Oca, Álava)», Veleia 29, 2012, 441-451.

Ortiz de Urbina, E., 2000, Las comunidades hispanas y el derecho latino, [Anejos de Veleia. Serie Minor 15] Vitoria-Gasteiz: UPV/EHU.

—,2009, «La representación de las élites locales y provinciales en los homenajes hispanos: la distinción honorífica pública y la intervención privada», en: Espacios, usos y formas de la epigrafía hispana en épocas antigua y tardoantigua: homenaje al Dr. Armin U. Stylow, [Anejos AEspA, 48], Mérida: Consejo Superior de Investigaciones Científicas e Instituto de Arqueología de Mérida, 227-245.

Ruiz-Alcalde, D. y Charquero, A. M., 2014, «El monumento funerario romano de la Torre de Sant Josep de Villajoyosa (Alicante). Nuevos datos y propuesta de restitución», Lucentum 33, 151-182. 